\title{
Towards a "Deep" Ontology for African Traditional Medicine
}

\author{
Armel Ayimdji ${ }^{1}$, Souleymane Koussoubé ${ }^{2}$, Laure P. Fotso ${ }^{3}$, Balira O. Konfé ${ }^{2}$ \\ ${ }^{1}$ Département de Génie Informatique, IUT de Douala, Douala, Cameroun \\ ${ }^{2}$ Laboratoire Africain d'Informatique et de Mathématiques Appliquées, IAI de Libreville, Libreville, Gabon \\ ${ }^{3}$ Departement d'Informatique, Université de Yaoundé I, Yaoundé, Cameroun \\ E-mail: ayimdji@gmail.com, skoussoube,obalira@yahoo.fr, laurepfotso@yahoo.com \\ Received August 12, 2011; revised September 29, 2011; accepted October 10, 2011
}

\begin{abstract}
The increasing interest on ontologies as the backbone technology for knowledge based systems implies the refinement of ontologies development methods. In this paper we propose a new approach to develop an ontology for African Traditional Medicine. The aim of our approach is to build a deep ontology by deepening concepts descriptions to formally represent all the semantics underlying the concepts used in African traditional medicine. We use a description logics language to formalize our approach.
\end{abstract}

Keywords: Primary Ontology, Deep Ontology, Hidden Aspects, Interpretations, African Traditional Medicine, Description Logics

\section{Introduction}

These last decades witness the rebirth of herbal-based treatments and Traditional Medicine (TM) across the world. Several factors can explain the increasing success of TM: it is a local medicine, less restrictive and very often cheaper than modern medicine (MM). Moreover, it is a credible alternative for low-income households. This has led to its recognition by $\mathrm{WHO}^{1}$ to work with researchers of its member countries to promote the use of TM for health care.

However, while in European and Asian countries TM is well documented, in most African countries, this knowledge is transmitted orally from father to son, from mother to daughter or from master to disciple. Therefore, as time goes by, this knowledge tends to deteriorate or to get lost when a practitioner of African traditional medicine (ATM) has no child or when none of his children keeps the interest on ATM. These are the main reasons behind the development of a knowledge based system (KBS) on ATM to store, maintain and facilitate knowledge sharing on ATM. Such a KBS should be ideally based on an ontology [1] in order to provide shared conceptualizations by formal descriptions of ATM concepts and relations between them.

\footnotetext{
${ }^{1}$ World Health Organization, http://www.who.int/
}

To our knowledge, the first and the only attempt to build an ontology for ATM was done in [2]. This first attempt has the merit of presenting some important concepts of the field, such as actors involved in ATM (healers, fetishists, soothsayers and magicians), treatment processes and general steps taken to cure a disease. However, this attempt is poor in concepts descriptions. The described ontology [3] looks more like a glossary than a deep analysis of ATM concepts. It mainly contains terms and their "primary" definitions. Furthermore, this ontology does not consider the deep semantics and specificities of ATM. Indeed, in ATM, the deep analysis of some concepts may reveal hidden aspects, appearing at a first glance as mystical considerations, but which can be captured and conceptualized in the ontology. In addition, staying at a lexical level in concepts descriptions allows only making lexical approximations between concepts of ATM and those of modern medicine.

Our work is different from [2] in that we propose a methodology for describing concepts with their various aspects and contexts of use. We provide a framework for the formal representation of the expertise of ATM practitioners and some "mystical" associated aspects. Thus, our approach allows, as much as possible, to give the possibility, on the one hand, to holders of knowledge (traditional healers) to express their knowledge on ATM, and on the other hand, to scientists (ethno-botanists, chemists, etc...) 
to complete this knowledge by integrating the various scientific interpretations that may be associated to each described concept. Our new approach constructs a "deep" ontology in ATM by considering different contexts of its use.

The rest of the paper is organized as follows. We briefly present ATM and motivate the need for its ontology in Section 2. In Section 3, we present Description Logics (DL), the formalism we use to formalize the ontology. Section 4 is devoted to the presentation of a formal framework to build a "deep" ontology for ATM. Finally in Section 5, we conclude and discuss how this work could be extended.

\section{African Traditional Medicine and Ontology}

The purpose of this section is not to fully describe ATM, but to present an overview which highlights the importance of the development of an ontology of this domain.

According to WHO, traditional medicine refers to the knowledge, skills and practices based on the theories, beliefs and experiences indigenous to different cultures, used in the maintenance of health and in the prevention, diagnosis, improvement or treatment of physical and mental illness. Traditional medicine covers a wide variety of therapies and practices which vary from country to country and region to region. In some countries, it is referred to as "alternative" or "complementary" medicine (CAM). Traditional medicine has been used for thousands of years with great contributions made by practitioners to human health, particularly as primary health care providers at the community level. TM/CAM has maintained its popularity worldwide. Since the 1990s its use has surged in many developed and developing countries. In Africa, $80 \%$ of the population depends on TM for primary health care. In many developing countries, $70 \%$ to $80 \%$ of the population has used one form or another of alternative or complementary medicine (e.g. Acupuncture ${ }^{2}$ ). Such expansion of TM in general and the ATM in particular, has encouraged the creation of dedicated scientific units ${ }^{3}$ conducting researches on medicinal plants, diseases and treatment processes based on these plants. Scientific publications of these research units do not reflect the actual level of the huge work done because most of the time, research results are only presented on ethnobotanical survey forms. These forms generally contain descriptive information such as specifications of the plants and their useful parts, names of the harvests, target diseases and medicine preparation methods. The

\footnotetext{
${ }^{2}$ http://www.who.int/topics/traditional medicine/en/

${ }^{3}$ E.g: IPHAMETRA (Institut de Pharmacopée et de Médecine Traditionnelle, Libreville, Gabon) is a research organism working on TM.
}

increasing number of publications in this field (e.g. [4-6]) as well as the variety of practices are such that improving the integration and the sharing of knowledge and information search on ATM is a real challenge. In addition, socio-cultural differences (different experiences, different training, different cultures, different needs, different perspectives, different languages or jargons, different contexts of use, etc ...) may cause real difficulties in ATM if different stakeholders do not have a common ontological basis.

The aim of introducing an ontology for ATM is to eliminate or at least reduce the conceptual and terminogical confusion and to walk towards a common and shared understanding in order to improve communication, sharing, interoperability and reusability. In order to satisfy these goals, ontologies such as [7] and [8] have been established in the field of medicine, and in particular an ontology for ATM has been proposed in [2]. Our work falls within this framework and aims at proposing a new approach for the construction of an ontology for ATM which considers the particularities and specificities of the domain. Uschold [9] notes that "no unified methodology is suitable for all ontologies, but different approaches are required for different circumstances". In order to set a possible matching between ATM concepts and those of the modern medicine, our approach allows translating ATM concepts in their semantics and therefore does not limit to lexical descriptions.

Before its processing by a computer, the ontology must be represented in a knowledge representation formalism. We use description logics which provide adequate inference mechanisms for hierarchical concepts descriions.

\section{Description Logics}

Description Logics (DL) [10] are knowledge representation formalisms used to describe concepts in a given field. A knowledge base (KB) described in DL comprises two components, the TBox and the ABox. The TBox introduces the terminology, i.e., the vocabulary of an application domain, while the ABox contains assertions about named individuals in terms of this vocabulary. The vocabulary consists of concepts, denoting sets of individuals (identified objects of the domain), and roles (binary relationships between individuals). In addition to atomic concepts and roles, all DL systems allow building complex descriptions of concepts and roles. Depending on provided operators, there are several DL languages, the "Attributive Language" (AL) being the minimal one. We summarize here the syntax and the semantics of some DL languages. 


\subsection{Syntax}

Concepts and roles are inductively defined from a set $N_{C}$ of concepts names (atomic concepts), a set $\boldsymbol{N}_{R}$ of role names (atomic roles) and a set of operators. In the folwing, unless otherwise stated, $\mathrm{A}$ and $\mathrm{B}$ are elements of $N_{C} ; \mathrm{r}$ and s are components of $N_{R} ; \mathrm{C}$ and D are concepts descriptions; $\mathrm{R}$ is a role description and $n$ is a positive integer. A description of a concept $\mathrm{X}$ and a role $\mathrm{R}$ in some DL languages is formed by the syntax of Figure 1.

The minimal language AL contains the atomic concept, the universal concept, the bottom concept, atomic negaon, intersection, value restriction and limited existential quantification: $A L=\{\mathrm{T}, \perp, \neg A, C \Pi D, \forall r \cdot C, \exists r\}$. As indicated by the syntax in Figure 1, we obtain more exessive languages if we add further constructors to AL. The added constructors determine the names of the obined languages:

- $\exists$ r.C: Full existential quantification, is indicated by the letter $\varepsilon$

- $\mathrm{C} \sqcup \mathrm{D}$ : The union of concepts, is indicated by the letter $\mathrm{U}$

- $\mathrm{C}$ : Full negation is indicated by the letter $\mathrm{C}$

- $\leq n r$, nr: unqualified number restriction is indicated by the letter $\mathrm{N}$

- $\leq n r$. C, nr. C: qualified number restriction, is indicated by the letter $\mathrm{Q}$

- $\mathrm{r}^{-}$: Inverse of role, is indicated by the letter I

Extending AL by any subset of the above constructors yields a particular AL-language. We name each AL-language by a string of the form $\mathrm{AL}[\mathrm{U}][\varepsilon][\mathrm{N}][\mathrm{C}][\mathrm{Q}][\mathrm{I}]$.

Hence, ALCQI is the language obtained from AL by adding full negation, qualified number restriction and Inverse of role.

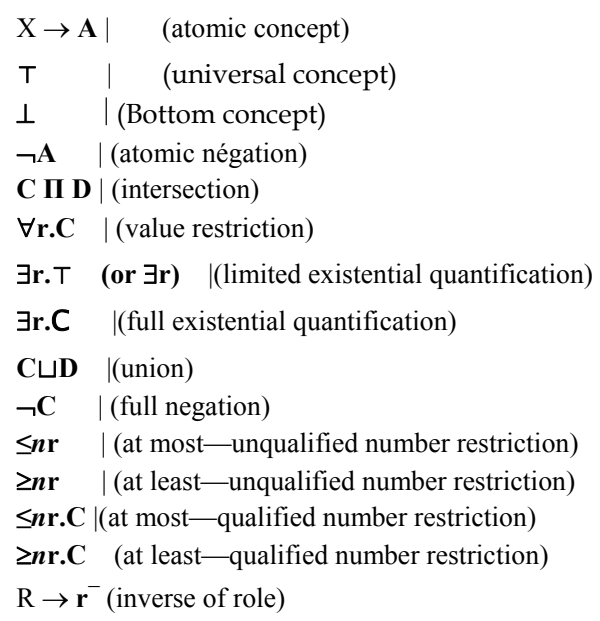

Figure 1. Syntax of concepts and roles descriptions in some DL.

\subsection{Semantics}

In order to define a formal semantics of concepts descriptions, we consider an interpretation $I$ that consists of a non-empty set $\Delta^{\mathrm{I}}$ (the domain of the interpretation) and an interpretation function.${ }^{I}$, which assigns to every atomic concept $A$ a set $\mathrm{A}^{\mathrm{I}} \subseteq \Delta^{\mathrm{I}}$ and to every atomic role $r$ a binary relation $r^{I} \subseteq \Delta^{I} x \Delta^{I}$. The interpretation function is extended to concept descriptions by the following inductive definitions in Table 1:

The signification of the sets in the "semantics" column in Table $\mathbf{1}$ is intuitive. For example, we have the following interpretations:

- $T$ is the whole domain, i.e. all the individuals in the domain.

- $\forall \mathrm{R} \bullet \mathrm{C}$ is the set of individuals who are related through $\mathrm{R}$ only to individuals satisfying $\mathrm{C}$.

- $\exists$ R. $\top^{\top}$ is the set of individuals related through R with other individuals of the domain.

- $\leq \mathrm{nR} \bullet \mathrm{C}$ is the set of individuals who are related through $\mathrm{R}$ to at most $\mathrm{n}$ individuals satisfying $\mathrm{C}$.

\section{Towards a Deep Ontology for African Traditional Medicine}

\subsection{A New Approach}

ATM practitioners (healers, soothsayers, fetishists ...etc.)

Table 1. Semantics of some concepts and roles descriptions in DL.

\begin{tabular}{|c|c|c|}
\hline $\begin{array}{c}\text { Concept } \\
\text { description }\end{array}$ & Semantics & Symbol \\
\hline$T^{I}$ & $\Delta^{\mathrm{I}}$ & \multirow{6}{*}{$A L$} \\
\hline$\perp^{\mathrm{I}}$ & $\varnothing$ & \\
\hline$(\neg \mathrm{A})^{\mathrm{I}}$ & $\Delta^{\mathrm{I}} \backslash \mathrm{A}^{\mathrm{I}}$ & \\
\hline$\left(\mathrm{C} \prod \mathrm{D}\right)^{\mathrm{I}}$ & $C^{I} \cap D^{I}$ & \\
\hline$(\forall \mathrm{R} \bullet \mathrm{C})^{\mathrm{I}}$ & $\left\{\mathrm{a} \in \Delta^{\mathrm{I}} \mid \forall \mathrm{b} .(\mathrm{a}, \mathrm{b}) \in \mathrm{R}^{\mathrm{I}} \rightarrow \mathrm{b} \in \mathrm{C}^{\mathrm{I}}\right\}$ & \\
\hline$(\exists \text { R.T })^{\mathrm{I}}$ & $\left\{\mathrm{a} \in \Delta^{\mathrm{I}} \mid \exists \mathrm{b} .(\mathrm{a}, \mathrm{b}) \in \mathrm{R}^{\mathrm{I}}\right\}$ & \\
\hline$(\exists \mathrm{R} \bullet \mathrm{C})^{\mathrm{I}}$ & $\left\{\mathrm{a} \in \Delta^{\mathrm{I}} \mid \exists \mathrm{b} .(\mathrm{a}, \mathrm{b}) \in \mathrm{R}^{\mathrm{I}} \wedge \mathrm{b} \in \mathrm{C}^{\mathrm{I}}\right\}$ & $\mathcal{E}$ \\
\hline$(C \sqcup D)^{I}$ & $C^{\mathrm{I}} \cup D^{\mathrm{I}}$ & $\mathrm{U}$ \\
\hline$(\neg \mathrm{C})^{\mathrm{I}}$ & $\Delta^{\mathrm{I}} \backslash \mathrm{C}^{\mathrm{I}}$ & C \\
\hline$(\leq n \mathrm{R} \bullet \mathrm{C})^{\mathrm{I}}$ & $\left\{\mathrm{a} \in \Delta^{\mathrm{I}}\left|\left\{\mathrm{b} \mid(\mathrm{a}, \mathrm{b}) \in \mathrm{R}^{\mathrm{I}}\right\}\right| \leq n \wedge \mathrm{b} \in \mathrm{C}^{\mathrm{I}}\right\}$ & \multirow{2}{*}{$Q$} \\
\hline$(\geq n R \bullet C)^{I}$ & $\left\{\mathrm{a} \in \Delta^{\mathrm{I}}\left|\left\{\mathrm{b} \mid(\mathrm{a}, \mathrm{b}) \in \mathrm{R}^{\mathrm{I}}\right\}\right| \geq n \wedge \in \mathrm{C}^{\mathrm{I}}\right\}$ & \\
\hline$(\leq n R)^{\mathrm{I}}$ & $\left\{\mathrm{a} \in \Delta^{\mathrm{I}}\left|\left\{\mathrm{b} \mid(\mathrm{a}, \mathrm{b}) \in \mathrm{R}^{\mathrm{I}}\right\}\right| \leq n\right\}$ & \multirow{2}{*}{$N$} \\
\hline$(\geq n R)^{\mathrm{I}}$ & $\left\{\mathrm{a} \in \Delta^{\mathrm{I}}\left|\left\{\mathrm{b} \mid(\mathrm{a}, \mathrm{b}) \in \mathrm{R}^{\mathrm{I}}\right\}\right| \geq n\right\}$ & \\
\hline$\left(\mathrm{R}^{-}\right)^{\mathrm{I}}$ & $\left\{(\mathrm{b}, \mathrm{a}) \in \Delta^{\mathrm{I}} \times \Delta^{\mathrm{I}} \mid(\mathrm{a}, \mathrm{b}) \in \mathrm{R}^{\mathrm{I}}\right\}$ & $I$ \\
\hline
\end{tabular}


make a number of assumptions that seems inconsistent with some principles of modern medicine. Terms used by those practitioners could sometimes refer to hidden aspects and therefore should not always be considered at the first degree. The semantics of the domain cannot be captured if these hidden aspects are not taken into consideration in the ontology. For example, consider the following two statements"

1) "Drink a calabash of the potion every morning."

2) "This plant should be collected by a young man, early in the morning, before the daybreak. Once the plant is collected, the collector must run straightly at home without stopping on the way, and the plant must be used immediately."

The first statement introduces the term "Calabash", a widely used container in ATM for therapeutic indications and conservation of healing potions. A "naive" or a purely lexical description of this concept in the ontology would restrict it to a container made from a fruit. However, a deeper observation of this indication shows that the concept "Calabash" in this context can refer to at least three different aspects: a fruit-based container, a dosage and/or a restriction (the medicine must be stocked exclusively in a calabash otherwise it would not be effective). This third aspect apparently superficial has a scientific basis because in many cases, the fruit forming the Calabash helps in a better preservation of the potion than a metal container where there can be a risk of corrosion or some chemical reactions between the liquid (potion) and the container.

The second statement describes the attitude to adopt when collecting some medicinal plants. It has been shown that plants requiring such an attitude contain essential oils for which the concentration is very high in the early morning and their volatility requires a very short delay between the collection and the usage. That is why some traditional doctors, without knowing this scientific explanation, recommend that such plants be collected by young men who can run fast.

These two situations show that in ATM, the ontologycal description of a concept has to go beyond the shallow conceptualization by structuring the ontology development process into two major steps: the first one consisting of primary descriptions of concepts and, the second one consisting of an incremental and iterative association of, if any, hidden aspects of the described concepts (see Figure 2).

- The first step builds a first ontology called the "primary ontology" where concepts are defined in a "naive" way; concepts are defined at the first degree ig-

${ }^{4}$ These statements were collected in real situations by researchers and collaborators of IPHAMETRA (Institut de Pharmacopée et de médecine Traditionnelle, Libreville, Gabon) with whom we collaborate. noring any eventual hidden aspects.

- The second step "deepens" concept descriptions in order to obtain the final ontology called the "deep ontology". This second step involves vari- ous specialists (ethnobotanists, chemists ...etc.) and aims at iteratively and incrementally introd- ucing the different hidden aspects (also called facets).

Figure 2 shows the process of the description of a concept in the proposed approach. First, the concept is primarily defined, and then, iteratively, the different stakeholders (specialists) involved in the ontology development process explore and add associated hidden aspects (facets). The following section aims at formalizing this process.

\subsection{Formalization}

We represent our ontology as a TBOX in which concepts are formally described using a description logics language, here $A L C$. It is easy to show (as in the chapter 2 of [10]) that from the semantic point of view $A L C$ also contains $\mathrm{U}$ (union) and $\varepsilon$ (full existential quantification):

$$
A L C \equiv A L C U \varepsilon
$$

Indeed, the semantics enforces the equivalences:

$$
\mathrm{C} \sqcup \mathrm{D} \equiv \neg(\neg \mathrm{C} \Pi \neg \mathrm{D}) \text { and } \exists \mathrm{R} \bullet \mathrm{C} \equiv \neg \forall \mathrm{R} \bullet \neg \mathrm{C}
$$

Hence, union and full existential quantification can be expressed using negation. Conversely, the combination of union and full existential quantification gives us the possibility to express negation of concepts.

Figure 3 shows the two ontologies $\left(O_{D}\right.$ and $\left.O_{P}\right)$ obtained during the development process described above.

In this figure, $C_{i}, i \in N^{*}$ represents a concept description

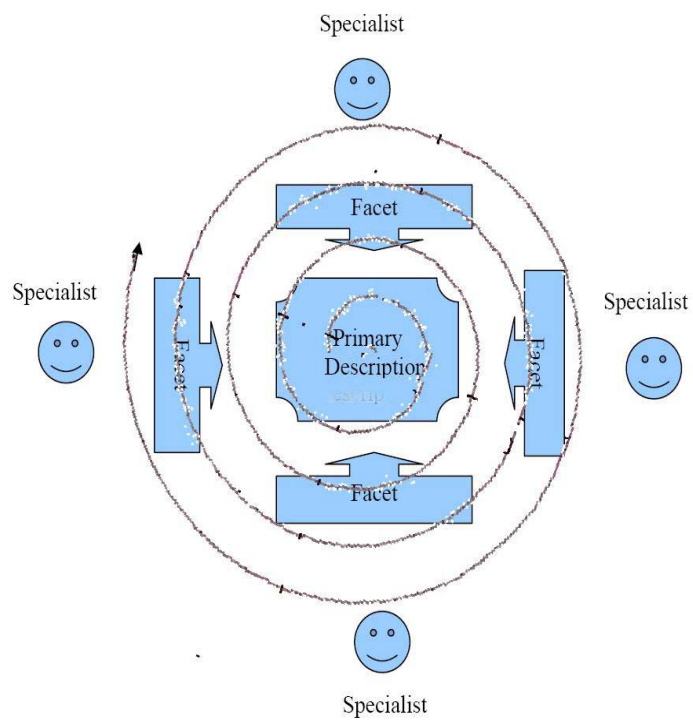

Figure 2. The deepening process of a concept primary description. 
in the deep ontology, the full description. To reach this, we must go through a primary description $C_{i}^{p}$ correonding to the definition of $C_{i}$ at the first degree ("naïve" description). This primary description is given by the exession:

$$
C_{i} \sqsubseteq C_{i}^{P}
$$

The set of these concept descriptions constitutes the primary ontology. Let $O_{P}$ be the primary ontology, then:

$$
O_{p}=\left\{C_{i} \sqsubseteq C_{i}^{P}, i=1 \cdots n, n \in N^{*}\right\}
$$

All the "facets" or aspects related to the various contexts of use of a concept are associated to each deep description of that concept. It should be noted that certain concepts (such as $C_{m}, m \in N^{*}$, in Figure 3) will not have any hidden aspects to formalize and no associated interpretation will be given. In this case, only the primary definition is taken into consideration. In order to associate to concepts their respective aspects, we create a primitive role which links concepts to interpretation. Let "ia" (an abbreviation for "interpreted as") this role. It will express the fact that a concept can be interpreted as some aspect. In general, if a concept $C_{i}$ can be interpreted as $j$ aspects $C_{i 1}^{a}, C_{i 2}^{a}, \ldots, C_{i j}^{a}$, then the following description is used to consider these aspects in the ontology:

$$
C_{i} \sqsubseteq \sqcup_{k=1 \ldots j}\left(\exists i a \bullet C_{i k}{ }^{a}\right)
$$

Then, from the expressions (1) and (3), we can give the expression of the deep description of each concept $C_{i}$ :

$$
\begin{gathered}
C_{i} \sqsubseteq C_{i}^{P} \sqcap\left(N I \sqcup\left(\sqcup_{k=1 \ldots j}\left(\exists i a \bullet C_{i k}^{a}\right)\right)\right) \\
i=1 \ldots n, n \in N^{*}
\end{gathered}
$$

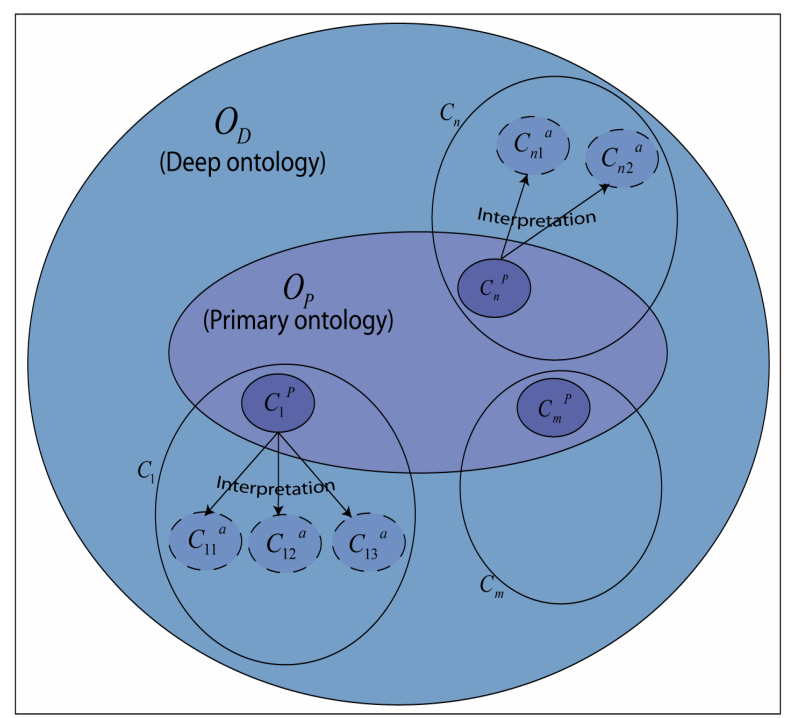

Figure 3. The primary ontology and the deep ontology. where NI (which stands for "Not Interpreted") is a special concept introduced to indicate the absence of hidden interpretation or hidden aspects. This special concept is used to restrict the concept description to the primary one when there is no hidden aspect found for a concept.

The set of these concepts descriptions constitute the deep ontology $O_{D}$ defined as:

$$
\begin{gathered}
O_{D}=\left\{C_{i} \sqsubseteq C_{i}^{p} \sqcap\left(N I \sqcup\left(\sqcup_{k=1 \ldots j}\left(\exists i a \bullet C_{i k}^{a}\right)\right)\right)\right. \\
i=1 \ldots n, n \in N^{*}
\end{gathered}
$$

\subsection{Formalization}

To illustrate this ontology development process, in the following two examples (example 1 and example 2), consider the two situations described in section $A$.

Example 1: "Drink a calabash of the potion every morning".

This statement can be considered as a therapeutic indication involving a Calabash. Here, to describe the concept "Calabash", the primary description reduces it to a fruit-based container.

Calabash $\sqsubseteq$ Container $\sqcap \forall$ madeFrom.Fruit

Then, the deep description extends the concept "Calabash" to the aspects of "Dosage" and "Restriction". By introducing a notation that precedes the name of each concept representing an aspect with "A_", we obtain:

Calabash $\sqsubseteq N I \sqcup \exists$ ia.A__Dosage $\sqcup \exists$ ia.A_Restriction

From the expressions (6) and (7) we can give the expression of the deep description of the concept Calabash:

Calabash $\sqsubseteq$ (Container $\sqcap \forall$ madeFrom. Fruit $) \sqcap$

(NI $\sqcup \exists$ ia.A__Dosage $\sqcup \exists$ ia.A_Restriction)

The development of expression (8) leads to:

$$
\begin{aligned}
\text { Calabash } \sqsubseteq(\text { Container } \sqcap \forall \text { madeFrom. Fruit } \sqcap N I) \\
\sqcup(\text { Container } \sqcap \forall \text { madeFrom. Fruit } \sqcap \\
\quad \exists \text { ia. A_Dosage }) \\
\sqcup(\text { Container } \sqcap \forall \text { madeFrom.Fruit } \sqcap \\
\quad \exists \text { ia.A_Restriction })
\end{aligned}
$$

The expression (9) shows that in the deep ontology, a Calabash is a container made from a fruit, or a container made from a fruit and is used to define a certain dosage, or a container made from a fruit and defines the obligation to respect a constraint (of preservation for example).

Example 2: "This plant should be collected by a young man, early in the morning, before the daybreak. 
Once the plant is collected, the collector must run straightly at home without stopping on the way, and the plant must be used immediately".

This second statement indicates the attitude to adopt when collecting the medicinal plant. Here, the challenge is twofold. First, we have to conceptualize the statement in order to formally represent the collection process of the plant. Second, we deepen the descriptions of the identified concepts in the conceptualization phase.

\section{a) Conceptualization phase}

This declaration of the traditional doctor highlights the importance of some concepts in the plant collection process: the specifications of the collector (young man), his attitude (run nonstop) and the period of the collection (early morning). We can use some formal concept to describe the situation. Generally, the specification of the collection process of a plant can be described as:

$$
\begin{aligned}
\text { Collection_Spec } & \sqsubseteq \forall \text { collector. Collector_Spec } \\
& \sqcap \forall \text { period.Period_Spec } \\
& \sqcap \forall \text { attitude.Attitude_Spec }
\end{aligned}
$$

By introducing more specific concepts, we refine the collection specification to fit the statement of our example:

$$
\begin{aligned}
& \text { Young_Man } \sqsubseteq \text { Collector_Spec } \\
& \text { Run_Non_Stop } \sqsubseteq \text { Attitude_Spec } \\
& \text { Early_Morning } \sqsubseteq \text { Period_Spec } \\
& \text { Thus, } \\
& \text { Collection_Spec1 } 1 \sqsubseteq \forall \text { collector. Young_Man } \\
& \sqcap \forall \text { period. Early_Morning } \\
& \sqcap \forall \text { attitude. Run_Non_Stop }
\end{aligned}
$$

So, Collection_Spec1 (the specification of the collection process of our example) is a particular kind of Collection_Spec.

We can easily imagine factual information (in an $\mathrm{ABOX}^{5}$ ) to model the studied statement:

Collection_Spec1(c1), Medicinal_Plant(p1), collection_ Process $(p 1, c 1)$ where Medicinal_Plant and collection_Process are respectively, the concept satisfied by all medicinal plants, and a relation linking each plant and his collection process.

\section{b) The deepening process phase}

The concept Early_Morning, specifying the appropriate collection time hides the aspect of essential oil while the concept Run_Non_Stop specifying the attitude of the collector hides the aspect of the volatility of an active ingredient. These hidden interpretations not explicitly stated by the healer can be formally represented in our

${ }^{5}$ The part of the knowledge based system containing assertions on individuals. An $\mathrm{ABOX}$ contains information like $\boldsymbol{C}(\boldsymbol{a})$ and $\boldsymbol{r}(\boldsymbol{a}, \boldsymbol{b})$ (i.e. respectively, the individual $\boldsymbol{a}$ satisfy the concept $\boldsymbol{C} ; \boldsymbol{a}$ is linked to $\boldsymbol{b}$ through the relation $\boldsymbol{R}$ ).
TBOX by the deepening process of concepts involved as follows:

$$
\begin{aligned}
\text { Run_Non_Stop } & \sqsubseteq \text { Attitude_Spec } \sqcap \\
& (\text { NI } \sqcup \exists \text { ia.A_A_Volatility_Constraint })
\end{aligned}
$$

Early_Morning $\sqsubseteq$ Period_Spec $\sqcap(N I \sqcup \exists$ ia.A_Essential_Oil_Concentration_Constraint)

\section{Practical Applications}

The principal aim of the deep ontology described in this paper is to standardize ATM concepts and thus, to standardize ATM practices while enabling context-aware applications development. Specifically, we think that research-based applications will find this ontology particularly helpful, joining what is done in the semantic Web. For example, in a Clinical Decision Support System (CDSS), this ontology will make it possible to software agents to have full access to information on patients and traditional doctors, including their beliefs and indigenous experiences, in order to infer patient-specific recommendations and to deeply explain these recommendations. Taking into account beliefs and indigenous experiences in this ontology-based CDSS will help traditional doctors to enable psychological considerations. Indeed, many people using MTA are not yet ready to accept that, behind some diseases known to be of unnatural origins, are sometimes scientific explanations. It is then necessary to harmonize diagnosis and treatment processes with their cultures and beliefs in order to put them in psychological states favoring recovery. At this level, the already existing ATM CDSS fail with respect to the WHO definition of ATM (see Section 2) because they don't consider beliefs and other metaphysic aspects. They just map physical signs to a set of recommendations.

We are now implementing an interactive ATM CDSS based on the deep ontology. The focus of our CDSS is the medical diagnosis and treatment recommendations. We have adopted a semantic web approach to model ATM knowledge coupled to a logic-based execution engine to reason over the ontology. We are using Protégé ontology editing environment to build our ATM ontology in OWLDL (Web Ontology Language-Description Logics). We also use Protégé OWL, the JENA API and SWI-Prolog to infer logic-based recommendations over the ontology. Figure 4 shows the current architecture (inspired from [11]) of our future ATM ontology-based CDSS.

The Rule Module will provide ATM experts an interface to specify decision logic rules, using a SWI-Prolog syntax. The rule authoring process is guided by the knowledge, relationships and constraints represented within the ATM ontology. The Rule Execution Module will execute SWI-Prolog rules (first transformed into JENA rules syntax) based on a patient instance (patient data acquired 


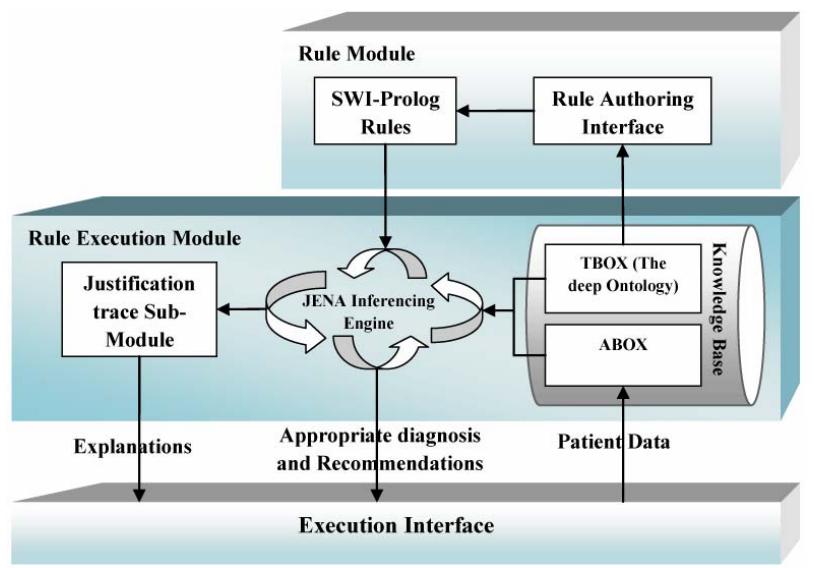

Figure 4. Architecture of the ontology-based ATM CDSS.

through the execution interface, based on the ontology) to infer diagnosis and recommendations. A justification trace of the inferred recommendations is then generated to explain the reasons for the proposed recommendations and the different interpretations if any, associated to some concepts appearing in the recommendations. In fact, the justification trace sub-module here is not only limited to explain the inference mechanisms, in addition it explains some concepts hidden aspects as described in the deep ontology. For instance, a recommendation can include the use of a "Calabash" and highlights a constraint warning. Then, the explanation clarifies the reason of the warning as a possible deterioration of the medicine if it is not kept in a Calabash.

\section{Concluding Remarks}

The development of an ontology for ATM based on interpretations of concepts has shown that certain aspects considered metaphysics or anecdotal at a first glance, sometimes hide rational and scientific interpretations that can formally be represented in the ontology to consider the richness of ATM. In this paper, using a DL language, we have proposed a framework for a formal representation of an ontology for ATM intended to be "deep" in that it differs from the ontology proposed in [2] by including all aspects that may be associated to various concepts used in ATM. Although we limited ourselves to ATM that we know better, our approach can be used for the development of an ontology for any TM. The Traditional Chinese Medicine for example, uses some concepts that deserve to be deeply analyzed to reveals possible hidden aspects.

Before a complete ontology for ATM can be built, there is need for a multidisciplinary team (including at least traditional healers, ethnobotanists, specialists of the ontological engineering) work to identify all relevant concepts in ATM that should be included. Once all the relevant concepts are identified, our proposed formal framework can be used to build a deep ontology for ATM as a basis for the perpetuation of knowledge and diverse scientific work done by authorized research units.

For a practical application, we have also sketched the architecture of a future ontology-based ATM CDSS.

\section{Acknowledgements}

Thanks to IPHAMETRA (Institut de Pharmacopée et de médecine Traditionnelle, Libreville, Gabon) where we found lot of information on ATM, especially to Maroufath Lamidi whose extensive comments on ATM practices greatly improve this paper.

\section{References}

[1] M. Uschold and M. Gruninger, "Ontologies: Principles, Methods and Applications," Knowledge Engineering Review, Vol. 11, No. 2, 1996, pp. 93-155. doi: $10.1017 / \mathrm{S} 0269888900007797$

[2] G. Atemezing and J. Pavón, "An Ontology for African Traditional Medicine," International Symposium on Distributed Computing and Artificial Intelligence 2008 (DCAI'08): Series: Advances in Intelligent and Soft Computing, Salamanca, Vol. 50, 2008, pp. 329-337.

[3] G. Atemezing, "African Traditional Medicine ontology," 2008. http://bioportal.bioontology.org/ontologies/40223

[4] S. A. Angone, H. Mathouet, A. Souza, L. Engone, F. Bivigou, C. E. M. Mba and M. Lamidi, "Quelques Plantes Utilisées en Médecine Traditionnelle Pour le Traitement de la Stérilité chez les Femmes au Gabon," Ethnopharmacologia, No. 43, 2009, pp. 52-58.

[5] L. P. Fotso, "Table of Entities and Attributes of Data Bases in MEDITRA (Knowledge Based on African Traditional Herbal Medicine)," Rapport de Recherche N 20, Université de Yaoundé I, Yaoundé, 1999.

[6] M. Lamidi, E. Ollivier, C. E. M. Mba, L. N. Ekekang and G. Balansard, "Enquêtes Auprès des Tradipraticiens Dans 3 Régions du Gabon sur des Plantes Utilisées Dans le Traitement des Diarrhées et des Helminthiases," Ethnopharmacologia, No. 32, 2003, pp. 39-51.

[7] Human Disease Ontology. http://www.obofoundry.org/ cgibin/detail.cgi?id=disease_ontology

[8] Infectious disease Ontology. http://www.obofoundry.Org/ cgibin/detail.cgi?id=infectious_disease_ontology

[9] M. Uschold, "Building Ontologies, Towards a Unified Methodology," in 16th Annual Conference of the British Computer Society Specialist Group on Expert Systems, 1996, pp. 16-18.

[10] F. Baader, et al., "The Description Logic Handbook: Theory, Implementation and Applications," Cambridge University Press, Cambridge, 2003.

[11] S. R. Abidi, S. S. Abidi, S. Hussain and M. Shepherd, 
"Ontology-Based Modeling of Clinical Practice Guidelines: A Clinical Decision Support System for Breast Cancer Follow-Up Interventions at Primary Care Set- tings," Studies in Health Technology and Informatics, Vol. 129, 2007, pp. 845-849. 